\title{
Space-VLBI phase-reference mapping and astrometry
}

\author{
J. C. Guirado ${ }^{1,2}$, E. Ros ${ }^{3}$, D. L. Jones ${ }^{4}$, J.-F. Lestrade ${ }^{5}$, J. M. Marcaide ${ }^{1}$ \\ M. A. Pérez-Torres ${ }^{6}$, and R. A. Preston ${ }^{4}$ \\ 1 Departamento de Astronomía, Universitat de València, 46100 Burjassot, Valencia, Spain \\ 2 Observatorio Astronómico, Universitat de València, 46100 Burjassot, Valencia, Spain \\ 3 Max-Planck-Institut für Radioastronomie, Auf dem Hügel 69, 53121 Bonn, Germany \\ 4 Jet Propulsion Laboratory, California Institute of Technology, Pasadena, California 91109, USA \\ 5 Observatoire de Paris-Meudon-CNRS, 92195 Meudon Principal Cedex, France \\ ${ }^{6}$ Istituto di Radioastronomia, CNR, Via P. Gobetti, 101, 40129 Bologna, Italy
}

Received 17 January 2001 / Accepted 9 March 2001

\begin{abstract}
We present $5 \mathrm{GHz}$ space-VLBI observations of the quasar pair B 1342+662/B 1342+663 that demonstrate the feasibility of phase-reference techniques using an antenna in space. The space-based data were taken by the satellite HALCA, of the space-VLBI mission VSOP. From residual (referenced) phases we derive an upper bound of 10 meters to the uncertainty of the spacecraft orbit reconstruction. An analysis of the phase-reference maps of the sources additionally suggests that the above mentioned uncertainty is likely not larger than 3 meters. With errors of this magnitude, HALCA is a useful tool for astrometric studies of close pairs of radio sources.
\end{abstract}

Key words. astrometry - techniques: interferometric - quasars: individual: B $1342+662$ - quasars: individual: B $1342+663$

\section{Introduction}

The relativistic jet theory for compact extragalactic radio sources assumes that most of the emission from quasars and AGNs comes from a central engine, predicted to be stationary at the microarcsecond ( $\mu$ as) level for standard cosmologies, ignoring opacity effects. In spite of the high precision provided by techniques such as VeryLong-Baseline Interferometry (VLBI) differential astrometry, the experimental status of this prediction is not satisfactory since only 3C 345 (Bartel et al. 1986) and $1038+528$ A/B (Marcaide et al. 1994) have been observed astrometrically with high enough precision to verify this stationarity. One of the largest contributors to the error budget of the observations above was the uncertainty inherent in the limited resolution of the radio source maps. The establishment of a global reference frame has a similar limitation: no matter how good the astrometric model may be, the lack of a reproducible point in the structure of the radio source, that may serve as reference, is the limiting factor of the precision. The latter is a necessary consequence of the nature of the observations: groundbased astrometry combines nearly microarcsecond-precise relative positions with only milliarcsecond-size features of the source structure.

Send offprint requests to: J. C. Guirado, e-mail: jcg@vlbi.daa.uv.es
Attempts to alleviate this situation should be directed to the extension of the VLBI phase-reference mapping and astrometry techniques to observations at the highest possible resolution, i.e., mm-VLBI and space-VLBI. The feasibility of differential astrometry at mm-wavelengths has already been demonstrated for a pair of sources with $5^{\circ}$ separation and high antenna elevations (Guirado et al. 2000). The launch in February 1997 of the HALCA (Highly Advanced Laboratory For Communication and Astronomy) satellite of the VLBI Space Observatory Program (VSOP; Hirabayashi et al. 1998) opened the study of radio sources at the highest possible resolution at cm-wavelengths. The potential capabilities of VSOP for astrometry are considerable, but, in practice, they are limited by the on-board command memory of the spacecraft and the uncertainties of the orbit reconstruction (Ulvestad 1999). However, an immediate advantage of space-VLBI observations is the improvement in the resolution of the image that makes the selection of a reference point rather straightforward and less affected by uncertainties.

In this paper, we explore the capabilities of VSOP for phase-reference mapping and astrometry by means of observations of the pair B 1342+662/B 1342+663 (Morabito 1984). This quasar pair is extremely favorable for the purpose: the separation of the sources is small enough so that both sources lie within the primary beam of the HALCA and the VLBA antennas. This particular configuration 


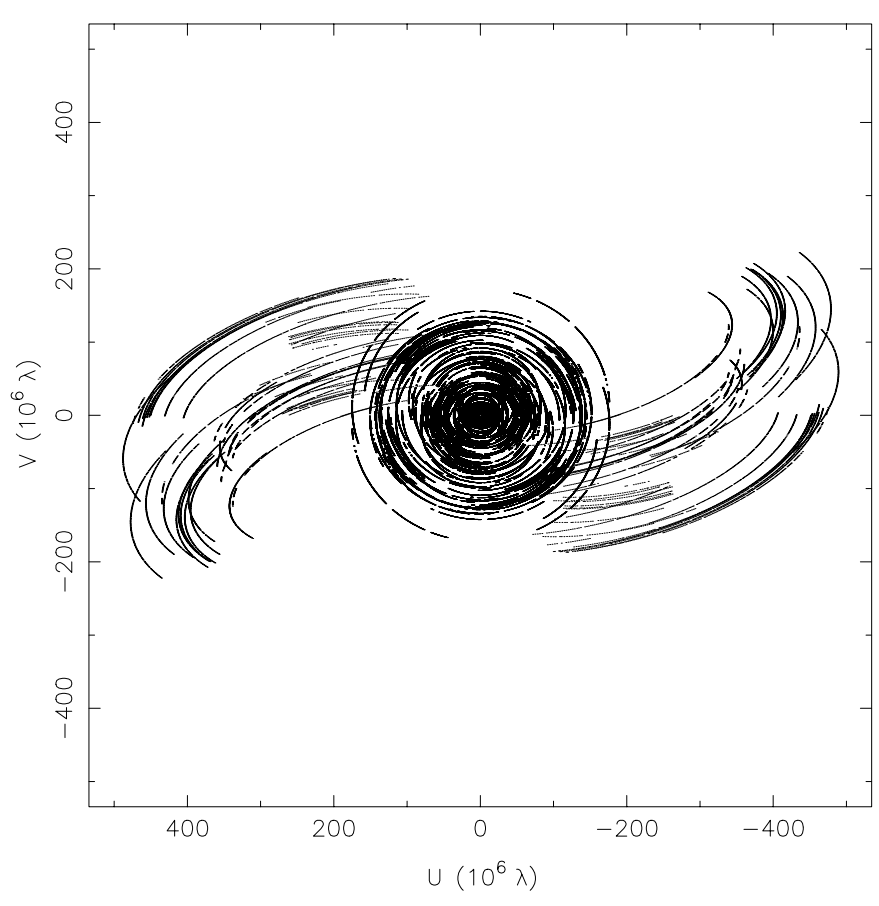

Fig. 1. $u v$-coverage of our space-VLBI observations. The denser set of points with $u v$-distances less than $200 \mathrm{M} \lambda$ corresponds to ground-based baselines. The outer tracks correspond to baselines to HALCA

permits an advantageous use of the phase-reference technique, where the interpolation of the observables in time, needed by switching schemes, can be avoided (Marcaide \& Shapiro 1983). Systematic errors (among them, orbit reconstruction errors) are thus largely cancelled. From the analysis of the phase-reference maps of the observed sources, upper bounds to the uncertainty of HALCA's orbit reconstruction can also be derived.

\section{Observations and data reduction}

We made observations of the radio sources B $1342+662$ and B 1342+663 on 1999 May 8 from 12:00 to 08:00 UT (next day). We used the complete VLBA (ten $25 \mathrm{~m}$ antennas), Effelsberg (100 m, Germany), and Kashima (34 m, Japan), along with the Japanese satellite HALCA ( $8 \mathrm{~m})$ at a frequency of $4.8 \mathrm{GHz}$, recording two intermediates frequency (IF) channels of $16 \mathrm{MHz}$ each, with a data rate of $128 \mathrm{Mb} / \mathrm{s}$. Five tracking stations received the astronomical data from HALCA through a Ku-band downlink, namely, Usuda (Japan), Robledo (Spain), Green Bank (USA), Tidbinbilla (Australia), and Goldstone (USA). We show in Fig. 1 the $u v$-coverage of our observations to emphasize the improvement in resolution provided by the baselines to HALCA.

Since the separation of B $1342+662$ and B $1342+663$ is only $0.08(4.8)$ on the sky, both sources were observed simultaneously by HALCA and the ground telescopes, except Effelsberg (whose antenna beam at $4.8 \mathrm{GHz}$ being 2.5 , switched between the sources; however, the switching time could not be well optimized for astrometric purposes and the data of baselines to Effelsberg had to be finally discarded). The data were correlated at the National Radio Astronomy Observatory (NRAO, Socorro, NM, USA). Two correlator passes were made, one at the position of each of the two sources. For each source, and using the NRAO Astronomical Image Processing System (AIPS), we made an a priori visibility amplitude calibration (using system temperatures and gain curves from each antenna), and a fringe fitting around the sky position used at correlation time. We optimized the 3 min-interval fringe solutions with the following iterative procedure: first, for each IF, we found fringe solutions using jointly delay and delay rates; second, using the previous solution as a priori and for each IF, we repeated the fringe fitting with narrow search windows in delay (50 ns) and delay rate $(100 \mathrm{mHz})$; finally, using the previous solution as a priori, we made a final fringe fitting for both IFs simultaneously to determine single-band delay, multi-band delay, and delay rate. The procedure described above was needed to overcome the lack of phase-calibration information for the satellite data, that prevented detections in several baselines to HALCA with a standard fringe fitting. As a result of our fringe fitting procedure, both sources were detected in all baselines, including those to HALCA.

For mapping purposes we transferred the data into the Caltech imaging program DIFMAP (Shepherd et al. 1995). We first performed several iterations of self-calibration in phase and amplitude using ground-based data alone; we proceeded further by adding data from HALCA to the ground-based data set. We used uniform weighting to obtain maps at maximum resolution (Fig. 2). It can be seen that using HALCA baselines, jet-like features are discernible towards the northeast in B $1342+662$ and towards the southwest for B $1342+663$, while at ground resolutions both sources appear unresolved.

\section{Results and discussion}

\subsection{Phase-reference mapping with HALCA}

Having direct detections of both sources for all baselines to HALCA allows us to calibrate the goodness of the phasereference technique. We performed a phase-reference analysis within AIPS (e.g. Beasley \& Conway 1995). Although we have for both sources healthy detections, we chose B $1342+663$ (the brighter source of the pair) to be the reference source. Therefore, the antenna phase calibrations of B $1342+663$ (free from structure effects by using a hybrid map of the source) were applied to the B $1342+662$ data.

The resulting phases of B $1342+662$, as said, referenced to the position of $\mathrm{B} 1342+663$, can be unambiguously tracked along the complete experiment (see Fig. 3; left panel). This is hardly a surprise for ground-ground baselines, but extremely important for baselines to HALCA: given two radio sources with a similar relative geometry to that of our pair, the uncertainties in HALCA's orbit determination do not prevent the prediction of the phases 

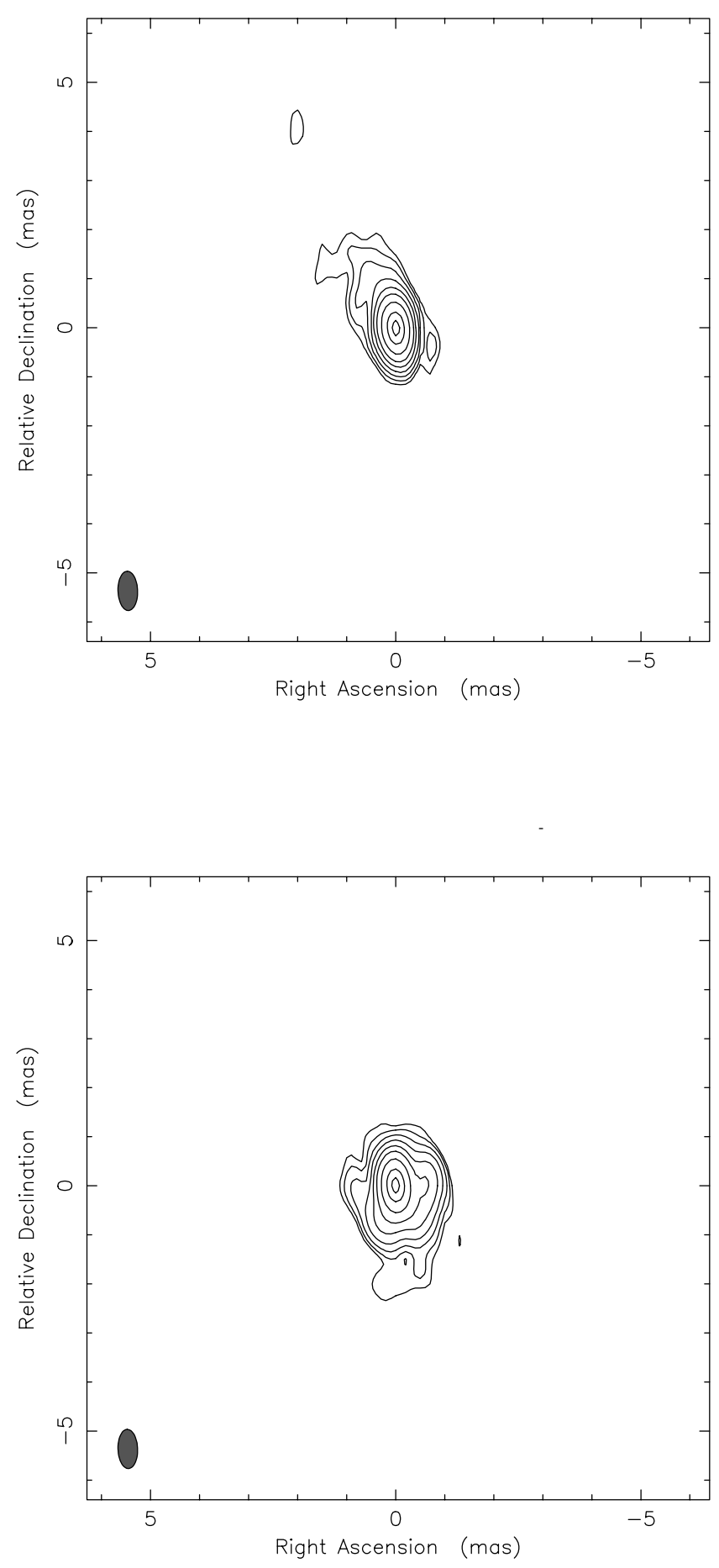

Fig. 2. $5 \mathrm{GHz}$ hybrid maps of $\mathrm{B} 1342+662$ (upper panel) and B 1342+663 (lower panel) at epoch 1999.35. Contours are -0.5, $0.5,1,2,4,8,16,32,64$, and $90 \%$ of the peak of brightness, $0.21 \mathrm{Jy} /$ beam (B 1342+662), and $0.42 \mathrm{Jy} /$ beam (B 1342+663). The restoring beam (shown at the bottom left corner of each map) is an elliptical Gaussian of $0.8 \times 0.4$ mas $\left(\mathrm{PA} 2^{\circ}\right)$

of one source from knowledge of the phases of the other. The phases in Fig. 3 (left panel) show a clear sinusoidal behavior, a result of the combination of the contributions of the structure of the target source, uncancelled effects of correlator model imperfections (necessarily angulardependent effects), and error in the relative coordinates of the two sources.

The error in the relative coordinates of the sources is by far the largest contributor to the non-zero referencephases. To show it, we obtained a phase-reference map of B 1342+662 (Fig. 4) by Fourier inversion of its (phasereferenced) visibilities. Both the offset with respect to the origin and, to a much lesser degree, the source structure (B 1342+662 is fairly compact) account for most of the systematic effects in the phases (see continuous line in Fig. 3; left panel). Once the structure contribution and the contribution of the offset position of B $1342+662$ are removed from the phases, the residual phases do not show significant systematic effects (Fig. 3; right panel). However, the root-mean-square (rms) of these residuals phases is $\sim 90^{\circ}$ on HALCA baselines, which provide an estimate of the inaccuracies of the satellite orbit reconstruction: at $5 \mathrm{GHz}$, and counting on the strong cancellation produced by the small separation of our sources, a rms of $\sim 90^{\circ}$ of the residual phases corresponds to $\sim 10 \mathrm{~m}$ uncertainties in HALCA's position. Further, the dispersion of the phases of some ground-ground baselines (e.g., baseline FD-SC in Fig. 3) is similar to that of the groundspace baselines, meaning that a significant part of the rms of the ground-space residual phases is due to "conventional" correlator uncertainties (e.g. geometry of the ground-based array and/or atmospheric effects over the ground station). Accordingly, our $10 \mathrm{~m}$ uncertainty estimate appears as a fairly conservative upper bound to the uncertainty in reconstructing HALCA's orbit. This conjecture can be verified, as shown below, from the astrometry carried out using different phase-reference maps of B $1342+662$.

\subsection{Differential astrometry}

The measurement of the position of the peak-of-brightness of the phase-reference image in Fig. 4 yields the differential correction to the a priori positions used in the correlator model. The peak-of-brightness of B $1342+662$ is located at position $-0.562 \pm 0.016$ mas and $1.383 \pm 0.008$ mas in right ascension and declination, respectively, where the quoted errors correspond solely to the uncertainty in determining the maximum of the brightness distribution in the image, based on the signal-to-noise ratio and the interferometric beam size.

To calibrate the contribution of the ground-space baselines in the relative astrometry, we made two additional phase-reference maps of B $1342+662$, namely, (1) a map using only ground-ground baselines (Fig. 5; left panel), and (2) a map using only ground-space baselines (Fig. 5; right panel). Each of the maps and offsets in Fig. 5 is to be compared with the image shown in Fig. 4 using all data. The ground-only phase-reference map of B 1342+662 shows, as expected, a point-like source, while the HALCAonly phase-reference map shows a clearer defined core-jet 

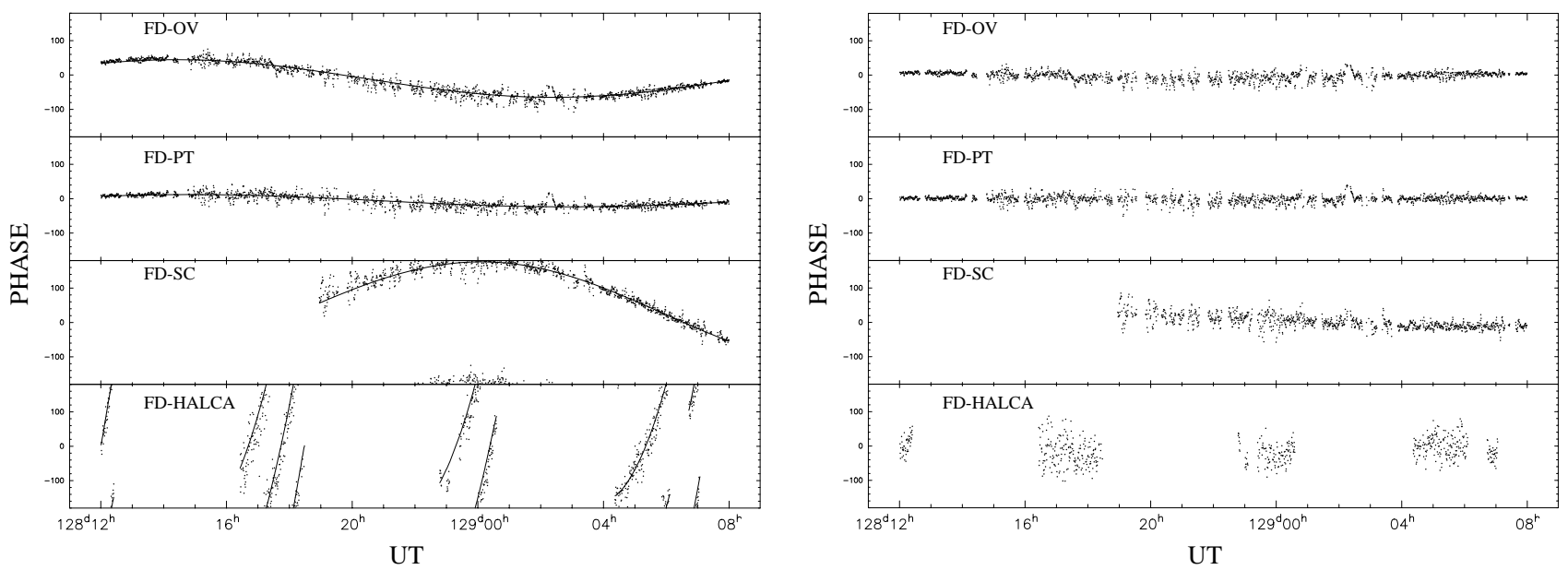

Fig. 3. Left panel: $5 \mathrm{GHz}$ phases of B $1342+662$ referenced to those of B $1342+663$ for a representative set of baselines. The continuous line corresponds to the phases derived from the phase-reference map of Fig. 4. Right panel: same as left panel after subtraction of the phases derived from the phase-reference map of Fig. 4 (continuous line in left panel). The symbols correspond to the following VLBA antennas: FD, Fort Davis; OV, Owens Valley; PT, Pie Town; SC, Saint Croix

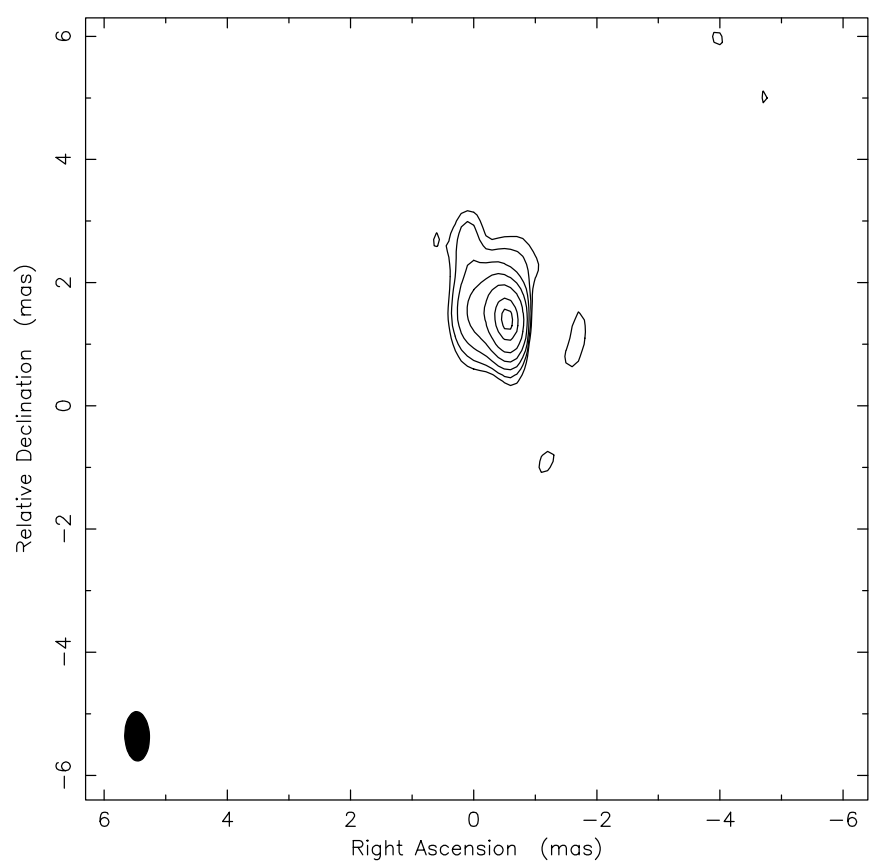

Fig. 4. $5 \mathrm{GHz}$ map of B $1342+662$ phase-referenced to B $1342+663$. Contours are $-2,2,4,8,16,32,64$, and $90 \%$ of the peak of brightness, $0.22 \mathrm{Jy} /$ beam. The restoring beam (shown at the bottom left corner) is an elliptical Gaussian of $0.8 \times 0.4$ mas $\left(\right.$ PA $2^{\circ}$ )

structure. The offsets of the three structures from the centers of the maps in Figs. 4 and 5 are extraordinarily similar. The differences between the offsets measured in any two maps of Figs. 4 and 5 are smaller than $70 \mu$ as. In particular, the difference in the relative position measured in the ground-only map (Fig. 5 left panel) with respect to that of the HALCA-only map (Fig. 5 right panel) may be interpreted as a rough estimate of the uncertainties in
HALCA's position. Such a difference is $\sim 60 \mu$ as. Again, taking into account the separation of 0.08 of our sources, we derive an estimate of the HALCA fractional baseline error of $\sim 2.110^{-7}$. For an average projected baseline of 12000 kilometers this error corresponds to an uncertainty in HALCA's position as small as $3 \mathrm{~m}$. This result is in agreement with that provided by Porcas et al. (2000), who estimated an error of about 2-5 $\mathrm{m}$ in HALCA's position for a pair of sources separated by $14^{\prime}$.

Finally, the offset of the peak-of-brightness in the phase-reference map of $\mathrm{B} 1342+662$ (Fig. 4), and the a priori coordinates of the correlator model can be transformed to the J2000.0 coordinates of the B $1342+662$ relative to those of $\mathrm{B} 1342+663$ at $5 \mathrm{GHz}$ : $\Delta \alpha=-0^{\mathrm{h}} 0^{\mathrm{m}} 22.720093 \quad \pm 0.000007$ $\Delta \delta=-0^{\circ} 3^{\prime} 45^{\prime \prime} .898617 \quad \pm 0$ '. 000050

where the quoted uncertainties have been enlarged to cover the differences between the relative separation obtained by using the two maps made with ground-ground only and ground-space baselines only, respectively. We notice that the astrometric uncertainty quoted above would not vary significantly from a sensitivity analysis of the different effects that contribute to the errors of the estimates of the relative coordinates. For pairs with such a small separation, most of this uncertainty comes from the reference point identification in the images (Marcaide et al. 1994; Rioja et al. 2000), whose contribution is already included in our estimate.

\section{Conclusions}

Our results show that phase-reference techniques can be applied to pairs of close radio sources using the HALCA satellite. The differential phase is unambiguously defined for ground-space baselines, with a rms of the residual phases of around $90^{\circ}$. Hence, radio sources with flux 

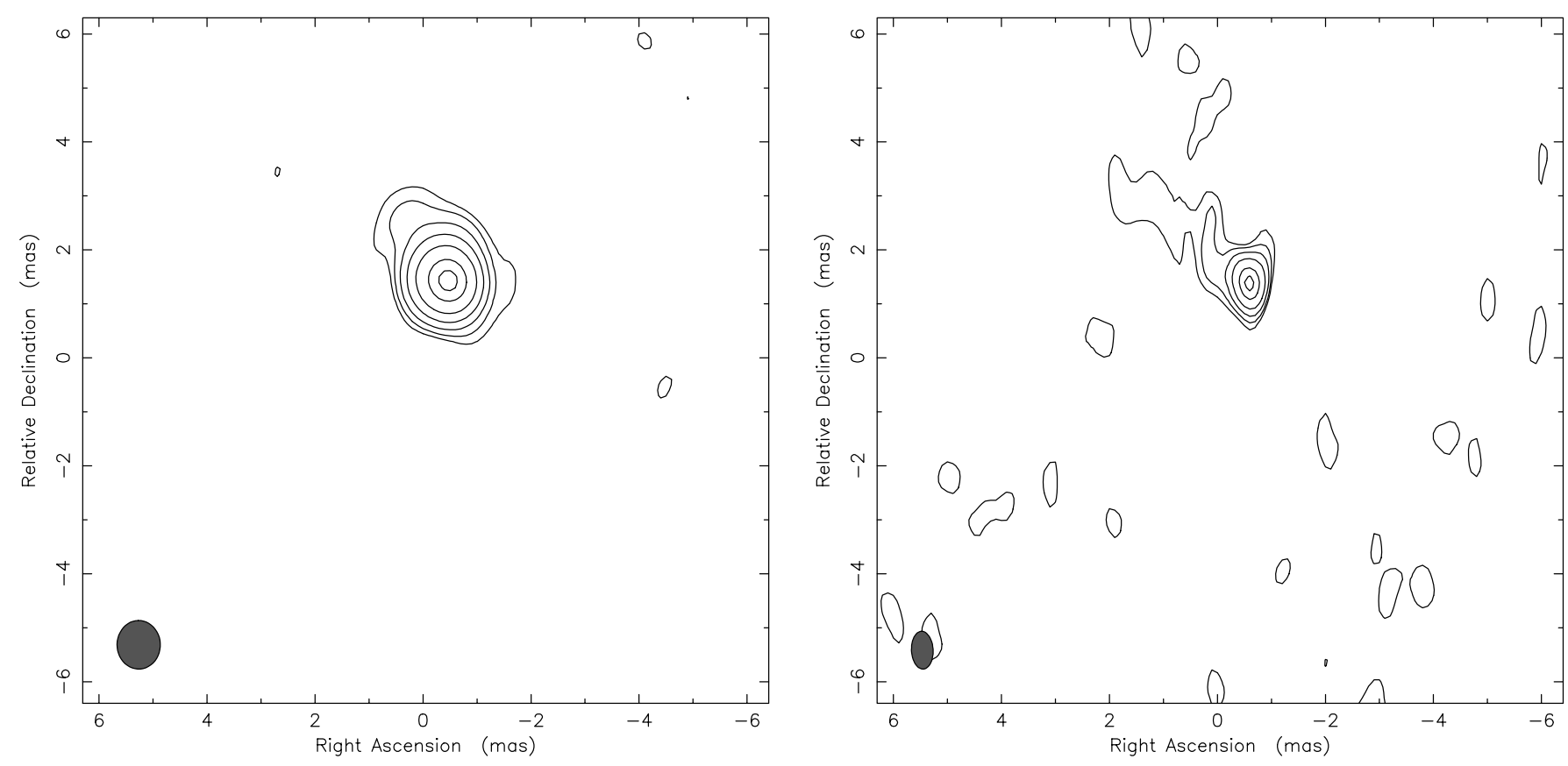

Fig. 5. Left panel: $5 \mathrm{GHz}$ map of B $1342+662$ phase-referenced to B $1342+663$ made with ground-ground data only. Contours are $-2,2,4,8,16,32,64$, and $90 \%$ of the peak of brightness, $0.25 \mathrm{Jy} /$ beam. The restoring beam (shown at the bottom left corner) is an elliptical Gaussian of $0.9 \times 0.8$ mas $\left(\mathrm{PA} 1^{\circ}\right.$ ). Right panel: same as upper panel but with ground-space data only. Contours are $-4,4,8,16,32,64$, and $90 \%$ of the peak of brightness, $0.26 \mathrm{Jy} /$ beam. The restoring beam (shown at the bottom left corner) is an elliptical Gaussian of $0.7 \times 0.4$ mas $\left(\mathrm{PA} 3^{\circ}\right)$

densities below HALCA's threshold detection can be observed if phase-referenced to nearby strong calibrators. We derive an upper bound of $10 \mathrm{~m}$ for the uncertainties of HALCA's position. These uncertainties could be even smaller, as suggested by the relative astrometry of different sets of phase-reference maps of our sources. With errors of this magnitude, HALCA is a useful tool for astrometry of close pairs of radio sources. The next generation of space-VLBI satellites will surely have a more accurate orbit determination (better than $10 \mathrm{~cm}$ if based on GPS receivers aboard; Ulvestad 1999). This accurate positions over the entire orbit will facilitate the successful application of space-VLBI to astrometry and geodesy.

Acknowledgements. We thank Jim Ulvestad for a constructive refereeing of the paper and Richard Porcas for his valuable comments. This work has been supported by the Spanish DGICYT grant PB96-0782. We gratefully acknowledge the VSOP Project, which is led by the Japanese Institute of Space and Astronomical Science in cooperation with many organizations and radio telescopes around the world. Research at JPL is carried out under contract with the National Aeronautic and Space Administration. The National Radio Astronomy Observatory is operated by Associated Universities Inc., under a cooperative agreement with the National Science Foundation.

\section{References}

Bartel, N., Herring, T. A., Ratner, M. I., et al. 1986, Nature, 319,733

Beasley, A. J., \& Conway, J. E. 1995, in ASP Conf. Ser. 82, Very Long Baseline Interferometry and the VLBA, ed. J. A. Zensus, P. J. Diamond, \& P. J. Napier, 327

Guirado, J. C., Marcaide, J. M., Pérez-Torres, M. A., \& Ros, E. 2000, A\&A, 353, L37

Hirabayashi, H., Hirosawa, H., Kobayashi, H., et al. 1998, Science, 281, 1825

Marcaide, J. M., \& Shapiro, I. I. 1983, AJ, 88, 1133

Marcaide, J. M., Elósegui, P., \& Shapiro, I. I. 1994, AJ, 108, 368

Morabito, D. D. 1984, in The Telecommunications and Data Acquisition Progress Report, 42-77 (Pasadena: JPL), 12

Porcas, R. W., Rioja, M. J., Machalski, J., et al. 2000, in Astrophysical Phenomena revealed by Space VLBI, ed. H. Hirabayashi, P. G. Edwards, \& D. W. Murphy (Sagamihara: ISAS), 245

Rioja, M. J., \& Porcas, R. W. 2000, A\&A, 355, 552

Shepherd, M. C., Pearson, T. J., \& Taylor, G. B. 1995, BAAS, 26,987

Ulvestad, J. S. 1999, in: ASP Conf. Ser. 180, Synthesis Imaging in Radio Astronomy II, ed. G. B. Taylor, C. L. Carilli, \& R. A. Perley, 513 